\title{
Efecto de la suplementación preparto con selenio levadura oral o selenato de bario parenteral en las concentraciones sanguíneas de selenio en vacas lecheras y sus crías
}

\author{
Effect of prepartum supplementation with selenized yeast or parenteral barium selenate \\ in blood selenium concentrations in dairy cows and their offspring

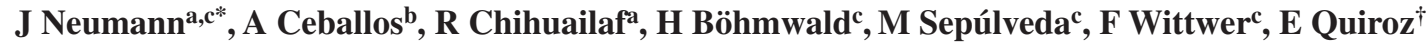 \\ ${ }^{a}$ Escuela de Medicina Veterinaria, Universidad Católica de Temuco, Temuco, Chile. \\ ${ }^{\text {b} G r u p o ~ d e ~ I n v e s t i g a c i o ́ n ~ C a l i d a d ~ d e ~ L e c h e ~ y ~ E p i d e m i o l o g i ́ a ~ V e t e r i n a r i a, ~ D e p a r t a m e n t o ~ d e ~ P r o d u c c i o ́ n ~ A g r o p e c u a r i a, ~}$ \\ Universidad de Caldas, Manizales, Colombia. \\ 'Instituto de Ciencias Clínicas Veterinarias, Universidad Austral de Chile, Valdivia, Chile.
}

\begin{abstract}
SUMMARY
In order to evaluate the effect of supplementation with two sources of selenium (Se) in blood and colostrum Se concentration in cows and their offspring, fourty three Holstein pregnant cows were used. Cows were randomly divided into three groups, and thirteen cows were supplemented one month before parturition with an organic source of Se ( $3 \mathrm{mg} / \mathrm{cow} /$ day p.o) (Se-yeast), another group of 15 cows was supplemented with a single dose of barium selenate (1 mg Se/kg LW SC) (Se-barium) and a third group of 15 cows remained un-supplemented (Control). Blood and colostrum Se concentrations of cows at calving and their offspring were similar between groups $(\mathrm{P}>0.05)$. However, blood activity of glutathione peroxidase increased in cows supplemented with Se-yeast $(307 \pm 21 \mathrm{U} / \mathrm{g} \mathrm{Hb})$ and Se-barium $(320 \pm 25 \mathrm{U} / \mathrm{g} \mathrm{Hb})$ compared to the control group $(221 \pm 14 \mathrm{U} / \mathrm{g} \mathrm{Hb})(\mathrm{P}<0.05)$. Similar findings are observed in the offspring. It is concluded that prepartum supplementation with Se as Se-yeast or Se-barium as used in this experiment does not alter blood and colostrum selenium concentrations in cows and their calves.
\end{abstract}

Key words: selenium, colostrum, blood.

\section{RESUMEN}

Con el objetivo de evaluar el efecto de la suplementación con dos fuentes de selenio en las concentraciones de selenio en calostro y sangre de vacas y de sus crías se suplementó un mes previo a la fecha estimada de parto a un grupo de 13 vacas (Se-levadura) con una fuente orgánica de selenio (3 mg/ vaca/día p.o), a otro grupo de 15 vacas (Se-bario) con selenato de bario (1 $\mathrm{mg} \mathrm{Se} / \mathrm{kg}$ peso vía SC, dosis única), y a otro grupo de 15 vacas permaneció sin suplemento (Control). No se encontraron diferencias en las concentraciones sanguíneas de Se en las madres y en las crías al momento del parto $(\mathrm{P}>0,05)$ y tampoco en las concentraciones de $\mathrm{Se}$ en calostro $(\mathrm{P}>0,05)$. Además se observó un incremento $(\mathrm{P}<0,05)$ de la actividad sanguínea de la enzima glutatión peroxidasa en las vacas suplementadas con levadura selenizada (307 $\pm 21 \mathrm{U} / \mathrm{g} \mathrm{Hb}$ ) y con selenato bario ( $320 \pm 25 \mathrm{U} / \mathrm{g} \mathrm{Hb})$ respecto de las controles (221 $\pm 14 \mathrm{U} / \mathrm{g} \mathrm{Hb}$ ), situación similar fue observada en las crías nacidas de estas vacas. Se concluye que la suplementación con Se-levadura o con Se-bario, en la forma empleada en este experimento, no modifican las concentraciones de selenio en sangre de las madres y sus crías así como en el calostro.

Palabras clave: selenio, calostro, sangre.

\section{INTRODUCCIÓN}

El elemento selenio es parte de los elementos fundamentales para la vida, donde la carencia acarrea problemas en la salud de los rumiantes, siendo la principal entidad asociada la enfermedad del músculo blanco (López y col 1997), y otras menos específicas como debilidad neonatal, retención de placenta, abortos, degeneración testicular, inmunosupresión y mastitis (Ceballos y Wittwer 1996). Por lo anterior, el Se ha sido materia de investigación en aspectos de nutrición animal.

Aceptado: 18.06.2015.

* Casilla 15-D, Temuco, Chile; jneumann@uct.cl

$\dagger \quad$ In Memoriam
El requerimiento nutricional de Se varía según la forma en que es ingerido, el criterio de adecuación que se emplee y la composición de la dieta (Underwood y Suttle 1999). Desde el punto de vista metabólico por medio del consumo en la dieta del elemento los rumiantes absorben el Se menos eficientemente y en forma más variable que los monogástricos (NRC 1983), debido a que una porción del selenito es reducido a formas insolubles por microorganismos en el rumen (Rasmussen y James 1994). Es por ello que se han desarrollado estrategias de suplementación basadas en productos inyectables (i.e selenito de sodio, selenato de bario), los que obvian el problema de la metabolización a nivel ruminal, y productos a base de selenio orgánico (incorporado en levaduras como selenio-metionina principalmente), estos son absorbidos 
más eficientemente que las formas inorgánicas. El Se es incorporado en los diferentes tejidos del organismo, en especial en la fracción proteica de los mismos. La excreción ocurre principalmente mediante la materia fecal, orina y leche (Ceballos y Wittwer 1996).

Igual que en la sangre y otros tejidos, la concentración de Se en la leche es afectada por su concentración en la dieta (Moeini y col 2009, Ceballos y col 2009). Es así que la concentración de Se en la leche de vaca varía según el consumo del mineral por parte del animal, aumentando cuando los animales son suplementados, encontrando incrementos en $0,16 \mu \mathrm{mol} / \mathrm{l}$ en leche posterior a suplementaciones orales con Se (Ceballos y col 2009). La transferencia de Se desde el plasma a la leche se realiza por un proceso de biorreducción, uniéndose a la fracción proteica de la leche, especialmente la caseína y en parte al suero y la grasa (Conrad y Moxon 1979).

El desarrollo y el crecimiento adecuado de fetos y terneros recién nacidos requieren de una adecuada transferencia de nutrientes por la placenta y la glándula mamaria. Es un hecho conocido que existen estrechas relaciones entre las concentraciones de Se en la sangre de terneros y sus madres al nacimiento (Kinkaid y Hodgson 1989, Abdelrahman y Kinkaid 1993). No obstante, en relación con la transferencia de Se al ternero existen divergencias, ya que algunos autores indican que es más eficiente por medio de la leche que transplacentaria (Underwood y Suttle 1999), mientras que otros sugieren que la vía transplacentaria es más importante (Enjalbert y col 1999). En relación con el metabolismo fetal del Se, estudios han determinado que las concentraciones de Se en el hígado incrementan entre los 120 y los 220 días de gestación, para luego decaer entre los días 221 y 270. Por lo demás, las concentraciones de Se sérico de vacas preñadas decaen durante los últimos 60 días de gestación (Abdelrahman y Kinkaid 1993).

Basado en lo anterior se propuso como objetivo de este trabajo evaluar el efecto de la suplementación un mes antes de la fecha esperada de parto con selenio orgánico vía oral o con selenato de bario vía parenteral en las concentraciones de selenio en sangre y calostro de vacas lecheras mantenidas a pastoreo y en la sangre de sus crías y además en la actividad de la enzima glutatión peroxidasa (GPx) en sangre de crías y madres.

\section{MATERIAL Y MÉTODOS}

\section{ANIMALES}

El estudio se realizó entre la época de invierno y primavera en el centro experimental Santa Rosa de la Universidad Austral de Chile (UACh), comuna de Valdivia, Chile (39 $38^{\prime}$ LS, $73^{\circ} 5^{\prime}$ LO). Se utilizaron 43 vacas de la raza Frisón Negro, clínicamente sanas al momento de realizar la exploración clínica y con una edad gestacional entre los 7-8 meses al inicio del ensayo.

\section{GRUPOS EXPERIMENTALES}

Las vacas fueron distribuidas en tres grupos experimentales designados como grupo Se-levadura $(n=13)$, grupo Se-bario $(n=15)$ y Control $(n=15)$. El diseño correspondió a bloques completos al azar, donde el criterio de bloqueo fue según la fecha probable de parto. Desde un mes previo a la fecha estimada de parto y hasta el parto las vacas del grupo Se-levadura fueron suplementadas diariamente por vía oral con una fuente orgánica de selenio (i.e levadura selenizada ${ }^{1}$ ). Cada vaca tuvo un consumo estimado de $3 \mathrm{mg} / \mathrm{vaca} /$ día, suministrados en forma de pellets de afrecho de trigo. Las vacas del grupo Se-bario recibieron un mes antes de la fecha estimada de parto una única dosis de selenato de bario ${ }^{2}\left(\mathrm{BaSeO}_{4}\right)$ en suspensión como producto de liberación lenta vía $\mathrm{SC}$, la dosis fue de $1 \mathrm{mg} \mathrm{Se} / \mathrm{kg}$ peso. El grupo control se mantuvo sin ningún tipo de suplementación con $\mathrm{Se}$, excepto lo recibido en la dieta base.

DIETA

El manejo general de estos animales se hizo en patio de alimentación donde se les suministró ensilaje de pradera (12 kg/vaca/día, Se =0,05 ppm) y heno de pradera (15 $\mathrm{kg} / \mathrm{vaca} /$ día, $\mathrm{Se}=0,04 \mathrm{ppm}$ ) distribuidas en dos raciones al día, además se le adicionó en el ensilaje 150 gr/vaca/ día de una mezcla mineral comercial preparto ${ }^{3}(\mathrm{Se}=20$ ppm). El ensilaje de pradera fue producido en el mismo predio en noviembre del año inmediatamente anterior, igual que el heno de pradera que también fue producido en el predio en enero del año que se realizó el estudio.

El consumo total de Se fue de 3,6 mg/día $(0,23 \mathrm{mg} / \mathrm{kg} /$ día) para los grupos Se-bario y Control, mientras que para el grupo Se-levadura fue de 6,6 mg/día (0,42 mg/ $/ \mathrm{kg} /$ día $)$.

A cada cría nacida se le suministró 4 litros de calostro (ordeñado desde su madre), por vía oral, mediante el uso de un biberón. El tiempo de administración fue dentro de las primeras 4 horas de nacido.

\section{MUESTRAS}

Se obtuvieron muestras de sangre tanto de las vacas como de sus crías. Las muestras de sangre $(5 \mathrm{~mL})$ fueron obtenidas por venopunción coccígea ventral en las vacas y yugular en los terneros. El sitio de obtención de muestras elegido fue de acuerdo con las facilidades de manejo de los animales. Las muestras fueron depositadas en tubos con heparina. La primera muestra fue obtenida previo a

Sel-Plex ${ }^{\circledR}$ (selenio: 1000 ppm y >95\% como orgánico). Alltech Inc, Nicholasville, KY, USA.

2 Deposel Multidose ® Novartis Animal Health Ltd, North Ryde, Australia.

3 Vetersal preparto ${ }^{\circledR}$, Veterquímica, Santiago, Chile. 
la suplementación, un mes antes de la fecha estimada de parto. En las vacas, el segundo muestreo fue realizado a las 24 horas postparto y en ese instante se obtuvieron también muestras de sangre de su cría. A las 24 horas postparto también se obtuvo de cada vaca una muestra de $50 \mathrm{~mL}$ de calostro mediante ordeña manual de los cuatro cuartos. Todas las muestras fueron congeladas a $-20^{\circ} \mathrm{C}$ hasta las posteriores determinaciones.

\section{ANÁLISIS DE CONCENTRACIÓN DE SE}

En las muestras de calostro y sangre se determinó la concentración de Se mediante espectrofotometría de absorción atómica con generación de hidruros. Las muestras, previa descongelación a $37^{\circ} \mathrm{C}$ fueron digeridas según un protocolo de 2 etapas y luego cuantificadas (Muñiz y col 2005). Para la digestión de calostro, en una primera etapa se pesaron aproximadamente $2,5 \mathrm{~g}$ de calostro previamente homogenizado. Se le adicionaron $2 \mathrm{~mL}$ de ácido nítrico $1 \mathrm{~mL}$ de peróxido de hidrógeno $30 \%$ y $2,5 \mathrm{~mL}$ de agua deionizada. Posteriormente fueron digeridas en un horno microondas ${ }^{4}$ siguiendo un programa de calentamiento de $10 \mathrm{~min}$ a $200^{\circ} \mathrm{C}$ y de mantenimiento por $10 \mathrm{~min}$ a $200^{\circ} \mathrm{C}$, luego se enfriaron $\mathrm{a}<50^{\circ} \mathrm{C}$, donde se agregaron $1,4 \mathrm{~mL}$ de ácido clorhídrico. Posteriormente las muestras fueron sometidas a un programa de $5 \mathrm{~min}$ a $130{ }^{\circ} \mathrm{C}$ y de mantenimiento por $10 \mathrm{~min}$ a $130{ }^{\circ} \mathrm{C}$. Luego fueron enfriadas a $<50{ }^{\circ} \mathrm{C}$ y se les agregó $0,4 \mathrm{~mL}$ de urea $(50 \% \mathrm{~m} / \mathrm{v})$ y se aforaron a $20 \mathrm{~mL}$ con agua deionizada.

La digestión de sangre se efectuó con $1 \mathrm{~mL}$ de sangre a la que se le adicionó $2 \mathrm{~mL}$ de ácido nítrico al $65 \%$, $1 \mathrm{~mL}$ de peróxido de hidrógeno al $30 \%$ y $2,5 \mathrm{~mL}$ de agua deionizada. Posteriormente fueron digeridas en horno microondas a $200{ }^{\circ} \mathrm{C}$ durante 20 minutos, luego se enfriaron $\mathrm{a}<50^{\circ} \mathrm{C}$ y se le adicionó $1,4 \mathrm{~mL}$ de ácido clorhídrico al $37 \%$. Luego se completó la digestión a $135^{\circ} \mathrm{C}$ durante 15 minutos. Posteriormente las muestras se enfriaron a $<50^{\circ} \mathrm{C}$, se adicionaron $0,4 \mathrm{~mL}$ de urea $(50 \% \mathrm{~m} / \mathrm{v})$ y finalmente se aforaron a $20 \mathrm{~mL}$ con agua deionizada.

La determinación de la concentración de Se fue realizada con un espectrofotómetro de absorción atómica ${ }^{5}$ a $196 \mathrm{~nm}$, con aditamento de generación de hidruros ${ }^{6}$. Para la calibración del equipo se contó con un patrón de Se con una concentración de $1.000 \mathrm{ppm}^{7}$, del que se obtuvo una dilución de 10 ppm. De esta última se elaboró una nueva dilución de 500 ppb, para así a partir de ella lograr los 3 estándares finales de 5,10 y $20 \mathrm{ppb}$, que junto con una muestra blanco se utilizaron para calibrar el equipo. Después

\footnotetext{
4 Milestone modelo Start D con rotor de 12 posiciones MRD/12S

5 Thermo Electron Solar S

6 Thermo Scientific VP100

7 Merck
}

de la lectura de 12 muestras consecutivas se procedió a recalibrar el equipo con un estándar y una muestra blanco.

\section{ANÁLISIS DE ACTIVIDAD SANGUÍNEA DE GLUTATIÓN PEROXIDASA}

La actividad sanguínea de glutatión peroxidasa (GPx; EC 1.11.1.9) se determinó mediante un método cinético modificado de la técnica de Paglia y Valentine (1967), utilizando un kit comercial ${ }^{8}$. El método está basado en una técnica cinética compuesta NADPH-dependiente, para lo que se utilizó un autoanalizador bioquímico. ${ }^{9}$

\section{ANÁLISIS ESTADÍSTICO}

Se realizaron análisis de normalidad (Shapiro-Wilk) y homocedasticidad (Bartlett) de los datos. Para las variables de concentración de Se en la sangre de vacas y sus crías, concentración de Se en calostro y actividad de GPx se establecieron diferencias entre grupos mediante un análisis de varianza (ANDEVA) para los datos paramétricos y se usó la prueba de Kruskal-Wallis para los datos con distribución no normal. La comparación y asociación entre las concentraciones de Se en sangre de las vacas y sus crías se estableció mediante el coeficiente de correlación de Pearson para los datos paramétricos y correlación de Spearman para los no paramétricos. Se utilizó un nivel de confianza de $95 \%(\mathrm{P}<0,05)$.

\section{RESULTADOS}

\section{CONCENTRACIÓN DE SELENIO EN SANGRE}

Las concentraciones medias de Se presuplementación entre grupos fueron similares y correspondieron a 1,04 $\mu \mathrm{mol} / \mathrm{L}, 1,15 \mu \mathrm{mol} / \mathrm{L}$ y $0,95 \mu \mathrm{mol} / \mathrm{L}$ para los grupos Se-levadura, Se-bario y Control. En el día 1 postparto la concentración sanguínea de Se en las vacas de los 3 grupos fueron similares a sus valores iniciales y de igual manera no hubo diferencia entre grupos (cuadro 1). Además, las concentraciones sanguíneas de Se en los terneros al nacimiento igualmente fueron similares en los tres grupos.

\section{CONCENTRACIÓN DE SELENIO EN CALOSTRO}

Las concentraciones de Se en el calostro no presentaron diferencias significativas entre los grupos $(\mathrm{P}>0,05)$. Los valores promedios $( \pm \mathrm{EE}$ ) obtenidos fueron $0,42 \pm 0,08$ $\mu \mathrm{mol} / \mathrm{L} ; 0,42 \pm 0,09 \mu \mathrm{mol} / \mathrm{L}$ y $0,31 \pm 0,04 \mu \mathrm{mol} / \mathrm{L}$ para los grupos Se-levadura, Se-bario y Control.

\footnotetext{
8 Ransel ®. Randox Laboratories Ltd. Crumlin, Irlanda del Norte.

9 Metrolab 2300 (Wiener Lab.)
} 
Cuadro 1. Concentraciones sanguíneas (promedio $\pm \mathrm{EE}$ en $\mu \mathrm{mol} / \mathrm{L}$ ) de selenio al parto en grupos de vacas suplementadas en el preparto con selenio levadura, selenito de sodio y control y en sus crías.

Blood concentration (mean $\pm \mathrm{SE}$ in $\mu \mathrm{mol} / \mathrm{L}$ ) of selenium at partum in groups of cows supplemented during the prepartum period with selenium yeast, barium selenite and control, and in their calves.

\begin{tabular}{lcccc}
\hline Grupo & Se-levadura & Se-bario & Control & P \\
\hline Vacas & $1,17 \pm 0,12$ & $0,96 \pm 0,13$ & $0,87 \pm 0,07$ & 0,181 \\
Crías & $1,33 \pm 0,13$ & $0,98 \pm 0,13$ & $1,10 \pm 0,08$ & 0,123 \\
P & 0,280 & 0,733 & 0,062 & \\
\hline
\end{tabular}

\section{ACTIVIDAD DE GLUTATIÓN PEROXIDASA}

La actividad sanguínea inicial de GPx fue similar entre grupos. A diferencia de las concentraciones de Se en sangre la actividad de GPx al parto fue superior en los grupos suplementados $(\mathrm{P}<0,05)$ respecto del grupo control, no presentando diferencias entre las dos fuentes de Se utilizadas en el estudio (figura 1). De igual manera la actividad sanguínea de GPx de los terneros nacidos de vacas suplementadas fueron superiores $(\mathrm{P}>0,05)$ respecto del grupo control.

\section{CORRELACIONES}

La concentración de Se en sangre en vacas al parto y del calostro recolectado el día del parto no evidenció una relación significativa en ninguno de los grupos, con valores de $r=-0,67(P=0,14)$ para el grupo Se-bario, $r=0,57(P=0,14)$ para el grupo Se-levadura y $r=0,45$ $(\mathrm{P}=0,31)$ en el grupo control.

La asociación entre las concentraciones de Se en la sangre de las vacas y de sus crías fue significativa solo en el grupo Se-bario $(r=0,88 ; \mathrm{P}<0,001)$; no así en los grupos Se-levadura $(\mathrm{r}=0,34 ; \mathrm{P}=0,28)$ y Control $(\mathrm{r}=0,34$; $\mathrm{P}=0,21$ ).

Para la asociación entre las concentraciones de Se en la sangre de las vacas y la actividad de GPx en sus crías no se evidenció correlaciones significativas, encontrando valores de $\mathrm{r}=-0,08(\mathrm{P}=0,80)$ para el grupo Se-levadura, $r=0,20(P=0,50)$ para el grupo Se-bario y $r=0,17$ $(\mathrm{P}=0,54)$ para el grupo control.

La asociación entre las actividades de GPx en la sangre de las vacas y sus crías presentaron valores de correlación moderada en el grupo Se-levadura $(r=0,59 ; \mathrm{P}=0,04)$,

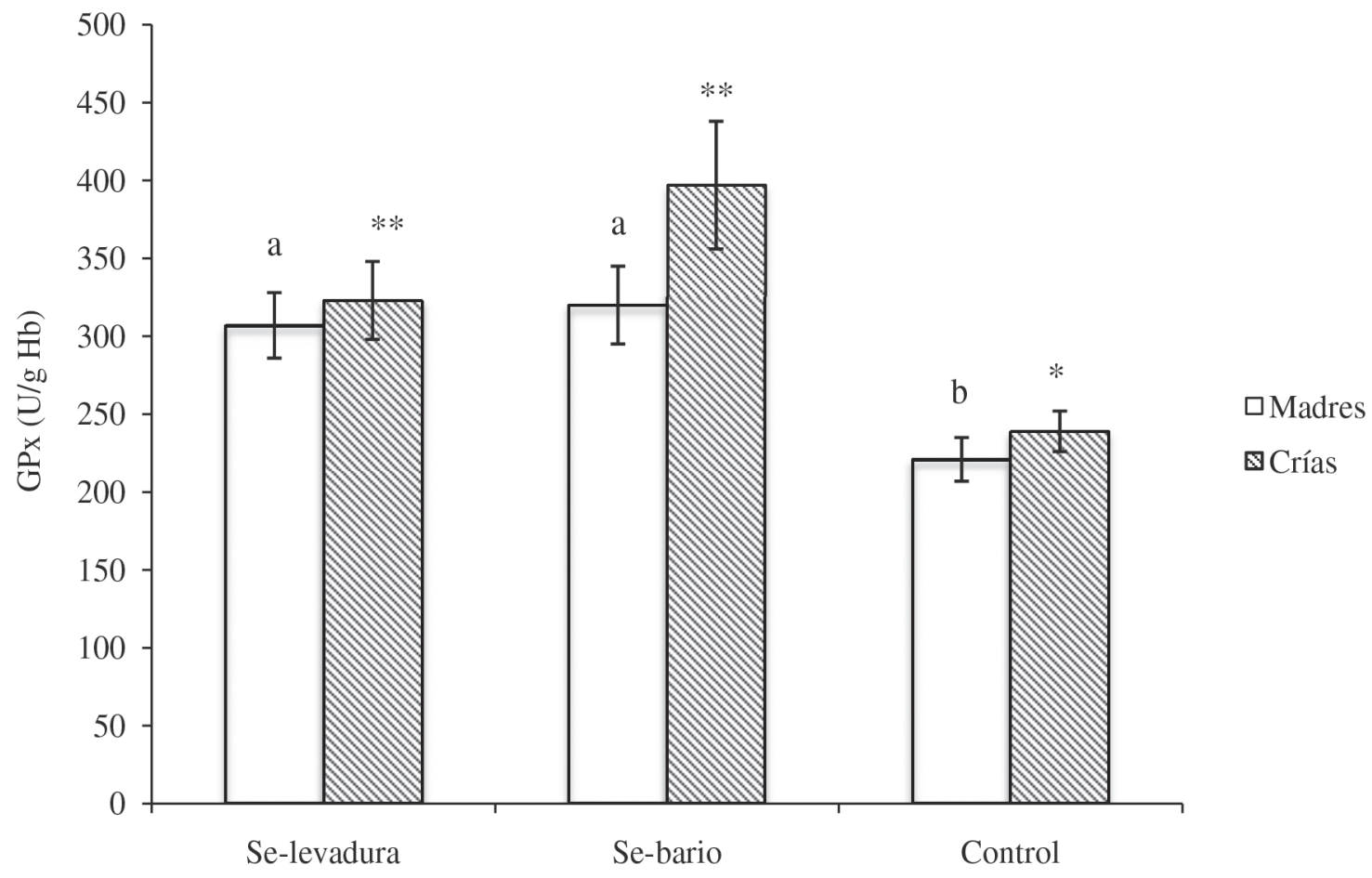

Figura 1. Actividad sanguínea de GPx (promedio \pm EE) al parto de vacas y sus crías suplementadas con dos fuentes de selenio y sin suplementación.

Blood GPx activity (mean \pm SE) at partum in blood of cows and their calves supplemented with two sources of selenium and without supplementation. 
mientras que en los otros dos grupos esta no fue significativa, con valores de $r=0,43(P=0,12)$ en el grupo Se-bario y de $\mathrm{r}=0,10(\mathrm{P}=0,72)$ en el grupo control.

\section{DISCUSIÓN}

En el ciclo de manejo de la vaca lechera el objetivo de suplementar con minerales en épocas estratégicas como lo es el periodo de secado tiene el propósito de ayudar a cumplir algunas metas, como es el caso de obtener crías bovinas nacidas con un buen estatus nutricional de Se, lo que tiene un impacto positivo en la salud de los terneros (Guyot y col 2007). Lo anterior puede contribuir a mejorar la función enzimática y la respuesta inmune, especialmente en épocas de estrés como sucede en animales de lechería en crecimiento (López y col 1997). A la luz de los resultados obtenidos en este estudio, la suplementación en el periodo de 30 días antes de la fecha esperada del parto con Se en las formas de levadura selenizada y selenato de bario tuvo un efecto positivo al aumentar la actividad sanguínea de GPx y, posiblemente, con ello la actividad antioxidante. La actividad de GPx en sangre se ha descrito como un indicador del balance metabólico de Se (Ceballos y Wittwer 1996, López y col 1997, Grace y col 2001) asociado principalmente cuando existen condiciones de aporte marginal o carente de Se donde se encuentran altas correlaciones entre las concentraciones sanguíneas de Se y la actividad de GPx (Ulrey 1987). De acuerdo con la clasificación de Ceballos y Wittwer (1996) los valores obtenidos de actividad de GPx (inclusive en el periodo preexperimental) son catalogados como adecuados (>130 $\mathrm{U} / \mathrm{g} \mathrm{Hb}$ ). En relación con otros valores publicados de GPX en la zona sur de Chile los valores de actividad de GPx encontrados son superiores a los reportados por Ceballos y col (1998), quienes obtuvieron en vacas preparto de ocho rebaños lecheros de la provincia de Valdivia un promedio de $161 \mathrm{U} / \mathrm{g} \mathrm{Hb}$ en relación con los $242 \mathrm{U} / \mathrm{g} \mathrm{Hb}$ previo al presente ensayo.

La sensibilidad de los fetos a los cambios en la suplementación maternal con minerales es influenciado por el transporte transplacentario y también por la inherente capacidad de los fetos para almacenar reservas (Gooneratne y Christensen 1989). El Se transferido por la madre se almacena en el hígado fetal, reserva que puede ser movilizada rápidamente permitiendo una actividad enzimática alta de GPx en las primeras semanas de vida (House y Bell 1994, Kirk y col 1995). Lo anterior podría explicar que se haya observado una mayor actividad de la enzima el primer día de vida de los terneros hijos de vacas suplementadas con Se.

En relación con las concentraciones sanguíneas de Se, los valores encontrados en el periodo preexperimental se encontraron en las categorías de bajo/marginal (grupos Selevadura y Control) y marginal (grupo Se-bario) de acuerdo con la clasificación de Ceballos y Wittwer (1996), y no alcanzan la categoría de suficiente de 1,2 $\mu \mathrm{mol} / \mathrm{L}$ (Jukola y col 1996), por lo que se debería esperar una respuesta positiva en términos de incrementos de concentraciones del mineral a la suplementación, ya que la respuesta a las suplementaciones es más consistente en estados de carencia $(<0,63 \mu \mathrm{mol} / \mathrm{L})$ (Pherson y col 1999). Sin embargo, en el presente ensayo no se obtuvieron diferencias en las concentraciones sanguíneas medidas al momento del parto. El consumo de levadura selenizada en los últimos 60 días de gestación puede tener un efecto benéfico en mejorar el estatus de Se en el ternero (Weiss 2005), pero la eficacia de la suplementación con Se levadura en dietas con una concentración de Se adecuada es incierta (Koenig y Beauchemin 2009). En el caso de este ensayo la suplementación se efectuó en los últimos 30 días de gestación, siendo probablemente poco tiempo para producir un incremento del Se en la sangre de las madres con la consecuente escasa transferencia al feto. Experiencias relacionadas con el efecto del tiempo de suplementación indican que la suplementación con selenito de sodio vía parenteral (44 mg) a las 8 y 4 semanas preparto tienen un efecto positivo en las concentraciones sanguíneas versus una suplementación única 4 semanas preparto (Pavlata y col 2003). No existen reportes publicados acerca del efecto de la suplementación en preparto con selenato de bario en las concentraciones sanguíneas de vacas lecheras. Como referencia cercana encontramos que Grace y col (2001) evidenciaron una respuesta positiva en las concentraciones sanguíneas de Se a los 41 días postsuplementación con selenato de bario en dosis de $1 \mathrm{mg} / \mathrm{kg}$, teniendo en cuenta que eran vacas con bajas concentraciones del elemento $(0,24 \mu \mathrm{mol} / \mathrm{L})$, lo que nos dice que efectivamente hay respuestas positivas en estados de carencia, a diferencia de lo presentado en nuestro estudio donde los valores son marginales y el aporte en la dieta es más alto que el ofrecido en el mencionado estudio. La suplementación con esta forma de Se tiene una duración efectiva de 12 meses (Wichtel 1998).

De acuerdo con lo obtenido en las correlaciones en los grupos suplementados se pueden encontrar dos situaciones, una en que la transferencia de Se al feto desde madres suplementadas con selenato de bario (formulación de depósito) es constante, asegurando una liberación constante del elemento, lo que se evidencia en la alta correlación positiva, a diferencia de lo observado en el caso de las crías nacidas de vacas que recibieron suplementación oral con Se orgánico en las cuales no se observó una relación entre concentraciones. Ello puede ser atribuido a que el consumo de selenolevadura involucra el consumo de Se-Met, la que es absorbida como tal e incorporada a cualquier proteína del organismo que contenga metionina, sirviendo como una reserva de Se corporal.

En relación con la transferencia de Se al calostro, se desconoce el mecanismo de incorporación del mineral en proteínas del calostro, encontrándose solo referencias en estudios efectuados en leche, estos indican que la incorporación del Se en las proteínas lácteas es menor 
para las formas inorgánicas, ya que al ser incorporado como Se-Cys su aporte a la leche es de solo 5,2 a 6,3\% (Calamari y col 2010). Los residuos del selenoaminoácido Se-Cys solo se encuentran en selenoproteínas y existen antecedentes de que la leche es escasa en selenoproteínas (Stagsted 2006). Los mayores incrementos de Se en leche corresponden generalmente a su inclusión en la caseína, bajo la forma de Se-Met, el que como selenoaminoácido puede ser incorporado inespecíficamente en proteínas que contengan metionina. Mayores concentraciones de Se en sangre no aseguran necesariamente una mayor transferencia al calostro. Pavlata y cols (2003) no encontraron que vacas con mayores concentraciones de Se en sangre tuvieran mayores concentraciones en calostro comparando vacas suplementadas con bajas concentraciones versus vacas con adecuado estatus de consumo de Se, por lo que el diagnóstico de carencia de Se por medición del elemento en el calostro no sería una buena herramienta diagnóstica.

La suma de factores, tiempo de suplementación y estatus previo de las concentraciones de Se en sangre influyen en la respuesta a las suplementaciones, lo que nos lleva a concluir que suplementando 30 días previo al parto con las formas de Selenato de bario y selenolevadura en condiciones de concentraciones sanguíneas de Se marginales no tiene un efecto en el incremento en las concentraciones del mineral en sangre o una diferencia en las concentraciones de Se en calostro respecto de vacas no suplementadas, siendo esperable, eso sí, un incremento en la actividad de la enzima GPx tanto de las madres como de las crías.

\section{AGRADECIMIENTOS}

Los autores expresan su agradecimiento a la Dirección de Investigaciones de la Universidad Austral de Chile por el financiamiento del Proyecto DID-UACH S-2007-52: "Suplementación con selenio en bovinos mantenidos a pastoreo: transferencia al calostro, leche y crías". Asimismo, a la empresa Alltech-Chile por financiar el proyecto Alltech: Selenium supplements "Transfer to milk and udder immunity in dairy cattle". Finalmente a los operarios encargados del manejo de las vacas en el fundo Santa Rosa de la Universidad Austral de Chile.

\section{REFERENCIAS}

Abdelrahman M, R Kinkaid. 1993. Effect of selenium supplementation of cows on maternal transfer of selenium to fetal and newborn calves. J Dairy Sci 78, 625-630.

Calamari L, F Petrera, G Bertin. 2010. Effects of either sodium selenite or Se yeast (Sc CNCM I-3060) supplementation on selenium status and milk characteristics in dairy cows. Liv Sci 128, 154-165.

Ceballos A, F Wittwer. 1996. Metabolismo del selenio en rumiantes. Arch Med Vet 28, 5-18.

Ceballos A, F Wittwer, P Contreras, H Böhmwald. 1998. Actividad sanguínea de glutatión peroxidasa en rebaños lecheros a pastoreo: variación según edad y época del año. Arch Med Vet 30, 13-22.

Ceballos A, J Sánchez, H Stryhn, J Montgomery, H Barkema, J Wichtel. 2009. Meta-analysis of the effect of oral selenium supplementation on milk selenium concentration in cattle. J Dairy Sci 92, 324-342.

Conrad H, R Moxon. 1979. Transfer of dietary selenium to milk. $J$ Dairy Sci 62, 404-411.
Enjalbert F, P Lebreton, O Salat, F Schelcher. 1999. Effects of pre or postpartum selenium supplementation on selenium status in beef cows and their calves. J Anim Sci 77, 223-229.

Gooneratne S, D Christensen. 1989. A survey of maternal and fetal tissue zinc, iron, manganese and selenium concentrations in bovine. Can J Anim Sci 69, 151-159.

Grace N, K Ankenbauer-Perkins, A Alexander, R Marchant. 2001. Relationship between blood selenium concentration or glutathione peroxidase activity, and milk selenium concentrations in New Zealand dairy cows. $N$ Z Vet J 49, 24-28.

Guyot H, P Spring, S Andrieu, F Rollin. 2007. Comparative responses to sodium selenite and organic selenium supplements in Belgian Blue cows and calves. Livestock Sci 111, 259-263.

House W, A Bell. 1994. Sulfur and selenium accretion in the gravid uterus during late gestation in Holstein cows. J Dairy Sci 77, 1860-1869.

Jukola E, J Hakkarainen, H Saloniemi, S Sankari. 1996. Effect of selenium fertilization on selenium in feedstuffs and selenium, vitamin $\mathrm{E}$ and beta carotene concentrations in blood of cattle. J Dairy Sci 79, 831-837.

Kinkaid R, A Hodgson. 1989. Relationship of selenium concentrations on blood of calves to blood selenium of the dam and supplemental selenium. J Dairy Sci 72, 259-263.

Kirk J, R Terra, I Gardner, J Wright, J Case, J Maas. 1995. Comparison of maternal blood and foetal liver selenium concentrations in cattle in California. Am J Vet Res 56, 1460-1464.

Koenig K, K Beauchemin. 2009. Supplementing selenium yeast to diets with adequate concentrations of selenium. Selenium status, thyroid hormone concentrations and passive transfer of immunoglobulins in dairy cows and calves. Can J Anim Sci 89, 111-122.

López M, M Miranda, J Hernández, C Castillo, J Benedito. 1997. Glutatión peroxidasa (GSH-Px) en las patologías asociadas a deficiencia de selenio en rumiantes. Arch Med Vet 29, 171-180.

Moeini M, H Karami, E Mikaelli. 2009. Effect of selenium and vitamin E supplementation during the late pregnancy on reproductive indices and milk production in dairy heifers. Anim Repr Sci 114, 109-114.

Muñiz O, R Domínguez, A Bermejo, P Bermejo, J Cocho, J Fraga, A Goris, A López, I Martínez, J Valledor, L Fernández. 2005. Selenium content and distribution in cow's milk supplemented with two dietary selenium sources. J Agric Food Chem 53, 9817-9822.

NRC, National Research Council, USA. 1983. Selenium in nutrition, Revised edition. National Academy Press, Washington, D.C., USA.

Paglia D, W Valentine. 1967. Studies on the quantitative and qualitative characterization of erythrocyte gluthatione peroxidasa. J Lab Clin Med 70, 158-169.

Pavlata L, J Prásek, A Podhorský, A Pechová, T Haloun. 2003. Selenium metabolism in cattle: maternal transfer of selenium to newborn valves at different selenium concentrations in dams. Acta Vet Brno 72, 639-646.

Pherson B, K Ortman, N Madjid, U Trafikowska. 1999. The influence of dietary selenium as selenium yeast or sodium selenite on the concentration of selenium in the milk of suckler cows on the selenium status of their calves. J Anim Sci 77, 3371-3376.

Rasmussen M, L James. 1994. Selenium metabolism in the rumen. In: Colegate S, Dorling P (eds). Plant Association Toxins. University of Arizona Press, Tucson, Arizona, USA.

Stagsted J. 2006. Absence of both glutathione peroxidase activity and glutathione in bovine milk. Int Dairy J 16, 662-668.

Ulrey D. 1987. Biochemical and physiological indicators of selenium status in animals. J Anim Sci 65, 1712-1726.

Underwood E, N Suttle. 1999. The mineral nutrition of livestock. $3^{\text {rd }}$ ed. CAB Publishing, Oxon, UK, Pp 421-475.

Weiss W. 2005. Selenium sources for dairy cattle. Proceedings Tri state dairy nutrition conference, Fort Wayne, USA, Pp 61-71.

Wichtel J. 1998. A review of selenium deficiency in grazing ruminants. Part 2: towards a more rational approach to diagnosis and prevention. $N Z$ Vet J 46 54-58. 